\section{Delirium postoperatorio: una consecuencia del envejecimiento poblacional}

\author{
EDUARDO VEGA P. ${ }^{1}$, CLAUDIO NAZAR J. ${ }^{1}$, MARCOS RATTALINO F. ${ }^{1}$, \\ JUAN PEDEMONTE T. ${ }^{1}$, MARCELA CARRASCO G. ${ }^{2}$
}

\section{Postoperative delirium among older people}

Delirium (acute confusional state) is a common and disabling complication among surgical older people. It is often underdiagnosed and undertreated. Its incidence varies by type of intervention and it is associated with several complications such as functional impairment, cognitive dysfunction, prolonged hospitalization and institutionalization. These increase hospitalization costs and the risk of death. There are precipitating and predisposing risk factors, which increase the susceptibility for postoperative delirium. This condition should be considered as a syndrome of epidemiological importance, which needs to be prevented or treated in a timely manner through a multidisciplinary intervention. The perioperative care of elderly patients involves different medical specialties and is a subject of general knowledge.

(Rev Med Chile 2014; 142: 481-493)

Key words: Delirium; Geriatrics; Postoperative complications.
'División de Anestesiología, Facultad de Medicina, Pontificia Universidad Católica de Chile. ${ }^{2}$ Departamento de Medicina Interna, Programa de Geriatría, Facultad de Medicina de la Pontificia Universidad Católica de Chile.

Recibido el 4 de julio de 2013, aceptado el 14 de octubre de 2013.

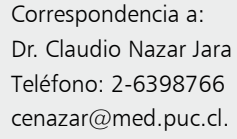

$$
\text { cenazar@med.puc.cl. }
$$

$\mathrm{E}$ n Chile existe un dramático envejecimiento poblacional, siendo alrededor de $8 \%$ la población mayor de 65 años con una proyección de $22 \%$ de la población total para el año $2050^{1}$. Entre 30 y $40 \%$ de esta población será sometida a algún procedimiento quirúrgico-anestésico, determinando una mayor frecuencia en la que estemos asistiendo a este tipo de pacientes ${ }^{2}$, siendo su cuidado perioperatorio transversal a diferentes especialidades y un tema de conocimiento general.

El procedimiento quirúrgico en el paciente mayor de 65 años pone en riesgo su vida y autonomía, pudiendo provocar cambios cognitivos en el postoperatorio, los cuales se dividen en delirium del despertar, delirium postoperatorio (DPO) y disfunción cognitiva postoperatoria. El DPO es el más severo, comparable a una falla de cualquier otro órgano posterior a una cirugía ${ }^{3}$. La definición actual de delirium está dada según el Diagnostic and Statistic Manual of Mental Disorders (DSM IV-TR) (Tabla 1$)^{4}$.

Existe otra entidad, el delirium subsindromal ${ }^{5}$, en la cual el paciente tiene uno o dos síntomas de delirium, pero no cumple con todos los criterios para su diagnóstico y no existe una progresión de los síntomas. Está asociado a peores resultados que aquellos que no lo presentan, pero mejor que quienes desarrollaron el cuadro de delirium completo $^{6-8}$. Al delirium subsindromal lo podríamos considerar como un "estado confusional de bajo grado", destacando la necesidad de clasificar la severidad de los estados confusionales".

\section{Métodos}

Se realizó una búsqueda electrónica en las bases de datos MEDLINE, EMBASE y Cochrane Library, con artículos publicados en inglés y español, desde el año 1985 hasta el año 2013, utilizando las palabras claves (MeSH database) delirium, postoperative complications, postoperative delirum, geriatric patient, antipsychotics y elderly surgical patient. Seleccionamos preferentemente metaanálisis, 
Tabla 1. Criterios diagnósticos de delirium según el DSM IV-TR (4)

A. Alteración de la conciencia (alteración de la atención), con disminución de focalizar, mantener o dirigir la atención

B. Cambio de las funciones cognoscitivas (memoria, orientación o lenguaje) o alteración de la percepción del ambiente, que no es explicado por demencia previa o en curso

C. Desarrollo en corto período de tiempo (horas a días) y tendencia a ser de curso fluctuante durante el día

D. Comprobación mediante historia, examen físico y de laboratorio que dicha alteración corresponde a un efecto fisiológico provocado de forma directa por una enfermedad médica, intoxicación o abstinencia de sustancia, uso de medicamentos, exposición a toxinas o combinación de estos factores

revisiones sistemáticas y trabajos prospectivos. Además, realizamos búsqueda secundaria de las referencias de los diferentes artículos revisados y se consideraron las recomendaciones de la Sociedad Americana de Medicina Intensiva, el Colegio Americano de Medicina Intensiva y del National Institute for Health and Care Excellence.

\section{Epidemiología y resultados asociados a delirium}

La incidencia de DPO es alrededor de $37 \%{ }^{10}$, teniendo relación directa con el envejecimiento. Es un síndrome de gran importancia epidemiológica ${ }^{11}$ ya que es frecuente, prevenible, habitualmente subdiagnosticado, subtratado y asociado a resultados adversos, aumentando los costos por paciente, tanto de la hospitalización (U\$2.000$3.000)^{12}$ como los anuales (U\$ 60.000) ) $^{13,14}$. Existen diferentes incidencias según la severidad de la enfermedad o cirugía y del método de evaluación empleado. Las mayores incidencias corresponden a grandes cirugías, como vascular ${ }^{15}$, abdominal mayor ${ }^{16}$, revascularización miocárdica ${ }^{17}$ y reparación de fractura de cadera ${ }^{18,19}$; siendo esta última la que presenta el mayor porcentaje de DPO (Figura 1). Dos tercios de los casos de DPO se presentan en los primeros tres días postoperatorios $^{20}$ y prácticamente entre $90 \%$ y $100 \%$ durante la primera semana ${ }^{21,22}$, teniendo una presentación transitoria ${ }^{23,24}$ y asociado a resultados adversos tanto intrahospitalarios ${ }^{25,26}$ como luego del alta (Figura 2). En un reciente meta-análisis de delirium intrahospitalario se demostró que los riesgos de mortalidad e institucionalización aumentan dos veces, mientras que el riesgo de demencia en más de 10 veces $^{27}$. En pacientes con diagnóstico preoperatorio de demencia, que sufren DPO, la velocidad de progresión del deterioro cognitivo puede acelerarse hasta en tres veces ${ }^{12}$. Sin embargo, hay que considerar que el delirium puede contribuir de forma directa o tan sólo ser un marcador de fragilidad. Independientemente de esto, el delirium es una alerta, hablándonos de un paciente en "riesgo" de presentar resultados adversos ${ }^{28}$.

Además, el delirium aumenta la duración de la hospitalización (5-10 días más) versus quienes no lo presentan ${ }^{29-31}$.

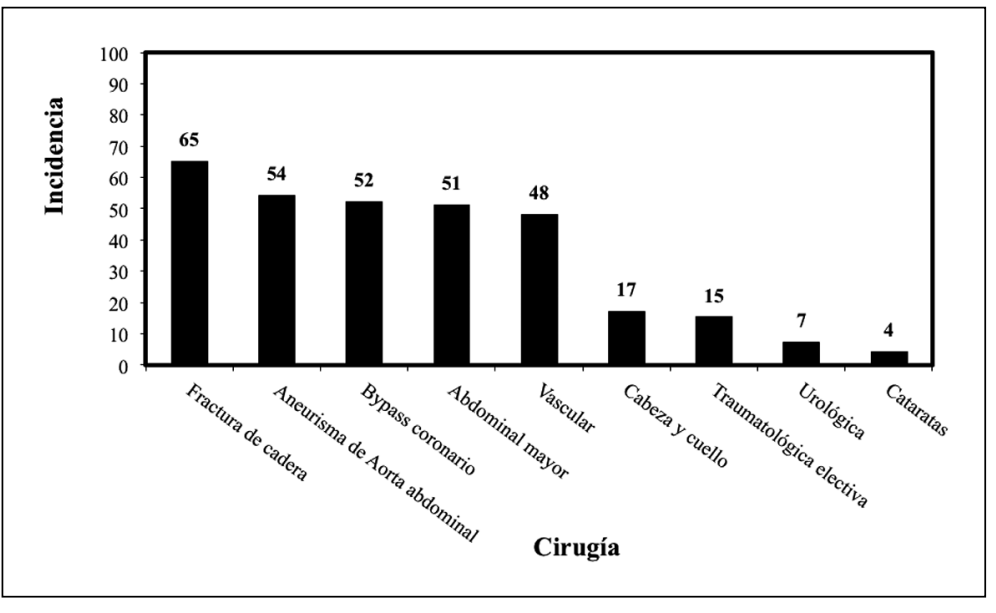

Figura 1. Incidencia de delirium en cirugías de alto y bajo riesgo. 


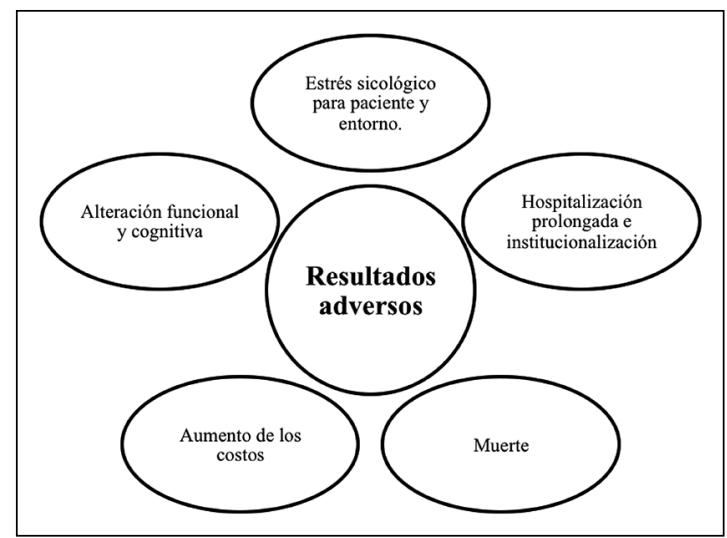

Figura 2. Resultados adversos asociados a delirium.

\section{Subtipos}

Según el comportamiento psicomotor ${ }^{32}$, existen tres subtipos: hipoactivo, hiperactivo y mixto. El hipoactivo es el más común, destacando una reducida atención y vigilancia, opuesto al subtipo hiperactivo, con un paciente agresivo e hipervigilante. En el subtipo mixto se alternan episodios de hipo e hiperactividad. Las prevalencias varían según los servicios clínicos, con alrededor de $20 \%$ correspondiente al delirium hiperactivo y $40-50 \%$ al mixto o hipoactivo. Por lo tanto, el menos frecuente (hiperactivo) es clínicamente obvio y de fácil diagnóstico, mientras que el más frecuente (hipoactivo) es el más subdiagnosticado y subtratado, asociándose con resultados más adversos ${ }^{33-35}$.

\section{Fisiopatología}

La etiología del DPO aún no está aclarada, existiendo diferentes mecanismos que juegan un rol preponderante en diversos escenarios clínicos $^{36}$. En la cirugía se liberan mediadores proinflamatorios (interleukinas 1, 6 y 8 y factor de necrosis tumoral $\alpha)^{37-39}$, los cuales en pacientes con aumento de la permeabilidad de la barrera hematoencefálica, como los pacientes añosos, la cruzan provocando daño neuronal (desde disfunción a muerte neuronal), produciendo la presentación clínica subsecuente como delirium de corta o larga duración, disfunción cognitiva postoperatoria o demencia ${ }^{40}$. Dicha neuroinflamación determinará una alteración de la neurotransmisión, con una disminución de la liberación de acetilcolina ${ }^{41}$ y un aumento en los niveles de dopamina ${ }^{42}$, ácido gamma amino butírico (GABA), serotonina $y$ norepinefrina ${ }^{43,44}$ (Figura 3 ).

Adicionalmente, el estímulo quirúrgico aumenta los niveles de cortisol plasmático, pudiendo originar o mantener el $\mathrm{DPO}^{45-47}$.

\section{Métodos diagnósticos}

El diagnóstico de delirium es clínico. El año 2010 un meta-análisis estudió 11 métodos clínicos objetivos de evaluación del delirium ${ }^{48}$, concluyendo que su elección dependería de la especialidad del examinador y del tiempo disponible para realizarlo. Sin embargo, la mejor evidencia sustenta el uso del Confusion Assesment Method

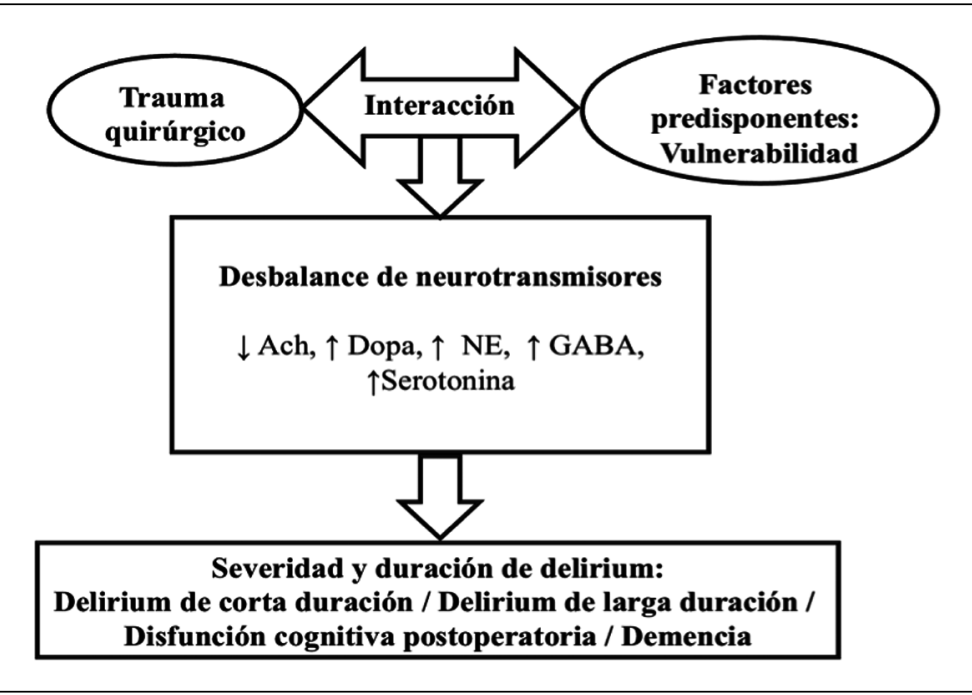

Figura 3. Mecanismo fisiopatológico de delirium. Ach: Acetilcolina, Dopa: Dopamina, NE: Norepinefrina, GABA: ácido gama amino butírico. 


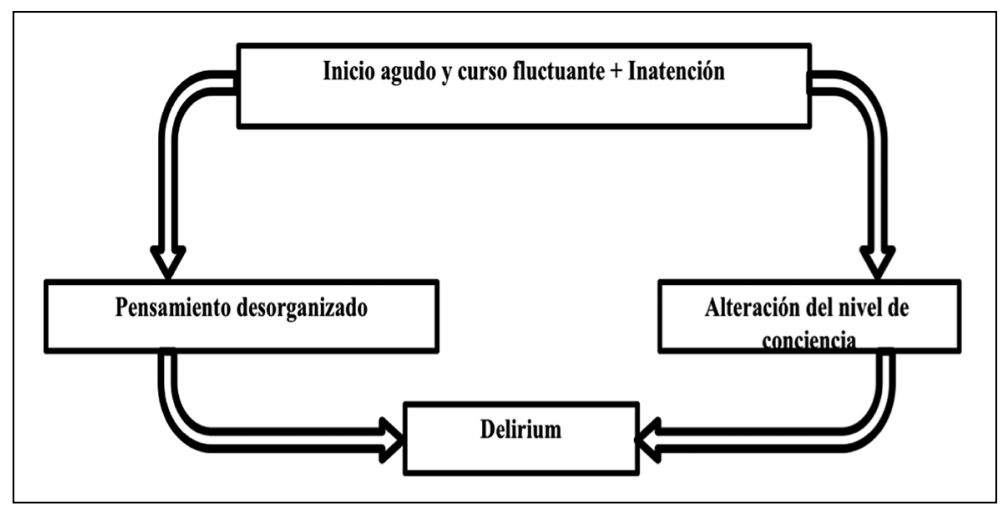

Figura 4. Algoritmo diagnóstico de delirium según $\mathrm{CAM}^{49}$.
$(\mathrm{CAM})^{49}$, tomando menos de cinco minutos en su realización por una persona entrenada ${ }^{50}$, con una sensibilidad de $86 \%$ y especificidad de $93 \%$. Los criterios diagnósticos del CAM se basan en el DSM-IV-TR, requiriendo obligatoriamente la presencia de los siguientes dos criterios: 1) inicio agudo y curso fluctuante y 2 ) inatención; más cualquiera de los siguientes dos criterios: pensamiento desorganizado o alteración del nivel de conciencia, para finalmente obtener o no, el diagnóstico de delirium (Figura 4).

En los pacientes de unidad de cuidados intensivos (UCI) los instrumentos diagnósticos son más recientes ${ }^{51}$, siendo el más estudiado el CAM $\mathrm{ICU}^{52-57}$.

Pese a los resultados asociados al DPO, no existe evidencia sólida que evalúe el impacto del tamizaje en los pacientes hospitalizados, concluyendo que el beneficio neto de su uso estaría definido por la edad, comorbilidades y severidad de la enfermedad actual ${ }^{58}$. Pese a esta carencia de evidencia, diversas organizaciones recomiendan su uso rutinariamente a todo paciente que ingresa al hospital, especialmente en pacientes con factores de riesgo para DPO. A pesar de los métodos diagnósticos objetivos, su aplicación sistemática no ha sido adoptada ${ }^{59,60}$, contribuyendo a que se mantenga como un problema subdiagnosticado y subtratado.

\section{Factores de riesgo}

Es conveniente dividirlos en dos grupos: predisponentes y precipitantes. El primero indica un paciente con mayor vulnerabilidad y baja reserva cognitiva para resistir diversas injurias. El segundo, son aquellos factores que precipitan el DPO en un paciente que presenta mayor o menor susceptibilidad ${ }^{61}$. Así, un paciente que posea muchos factores predisponentes necesitará unos pocos factores precipitantes para desarrollar DPO, mientras que el paciente con bajo número de factores predisponentes necesitará de un mayor número o una mayor severidad de factores precipitantes para presentar esta complicación.

Diversos modelos predictivos se han propuesto en base a estos factores de riesgo ${ }^{62-67}$, existiendo el problema de poder adaptar sólo uno a una población tan heterogénea como los pacientes quirúrgicos. Una reciente revisión ${ }^{68}$ concluye que la clave para predecir el DPO es tomar en consideración tres variables: rapidez del desarrollo de la injuria ${ }^{69}$, severidad de ésta ${ }^{70}$ y estado de salud preoperatorio (Figura 5). Este último envuelve múltiples factores como edad avanzada ${ }^{70,71}$, comorbilidades importantes, demencia preexistente ${ }^{72}$, depresión

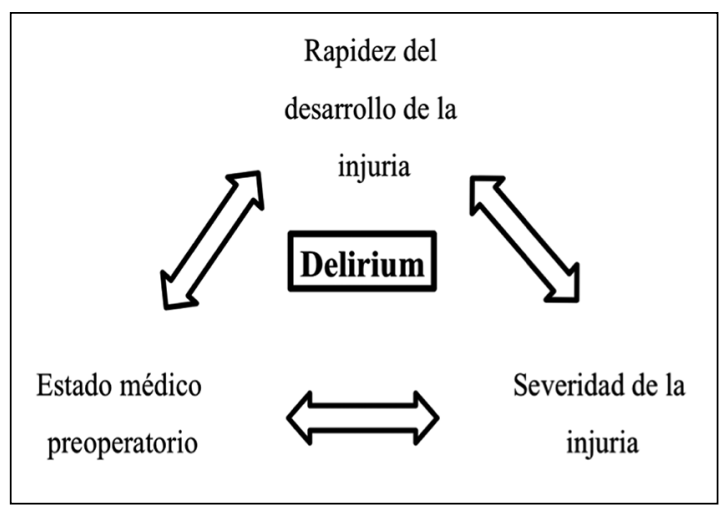

Figura 5. Predicción del delirium postoperatorio según los 3 factores principales involucrados en su génesis ${ }^{68}$. 
activa $^{72}$, alteración funcional ${ }^{69,70}$ y alteraciones de natremia, glicemia, nitrógeno ureico plasmático y proteína $\mathrm{C}$ reactiva ${ }^{71,72}$.

\section{Medidas de prevención}

Debido a los resultados adversos del DPO se ha propuesto realizar medidas preventivas en todos los adultos mayores con factores precipitantes, consistentes en medidas no farmacológicas y farmacológicas.

\section{Medidas no farmacológicas}

Existe una sólida evidencia demostrando su efectividad, conteniendo $\operatorname{costos}^{73} \mathrm{y}$ evitando adicionar nuevos fármacos.

El primer estudio realizado en población con patología médica evaluó seis factores de riesgo (alteración cognitiva, deprivación de sueño, inmovilidad, alteración visual o auditiva y deshidratación) e implementó sus respectivas intervenciones, disminuyendo el riesgo relativo de delirium en $40 \%{ }^{74}$, lo cual fue reproducido en población sometida a cirugía abdominal mayor, con $0 \%$ de DPO en grupo intervención versus $16,7 \%$ en el control ${ }^{75}$.

Otro modelo es la evaluación activa del equipo de geriatría. Marcantonio ${ }^{76}$ realizó el primer estudio aleatorio en pacientes mayores sometidos a cirugía de fractura de cadera, enfocado en diez medidas preventivas (control del dolor, reducción de medicamentos psicotrópicos y drogas anticolinérgicas, aporte de oxígeno, balance hidroelectrolítico, nutrición, mantención de la función intestinal y micción, prevención de complicaciones postoperatorias, movilización precoz, estimulación cognitiva y tratamiento precoz de agitación y delirium), logrando una reducción del riesgo relativo de DPO de $36 \%$ y, específicamente, de delirium severo en más de $50 \%$.

Esta aproximación diseñada por un equipo multidisciplinario puede prevenir alrededor de un tercio los casos de $\mathrm{DPO}^{77}$, siendo eficaz tanto en el sistema privado como público de atención en salud ${ }^{78}$. Sin embargo, el peso específico de cada intervención se desconoce (Tabla 2).

En pacientes de UCI, la temprana movilización y el manejo protocolizado de sedación y ventilación mecánica ${ }^{79,80}$ han reducido los días de asistencia ventilatoria y hospitalización en la unidad, y las tasas de delirium, logrando una mayor independencia física luego del alta ${ }^{81}$.

Lamentablemente, la aplicación de estas medidas es poco frecuente, pese a la sólida evidencia existente.

\section{Medidas farmacológicas}

Incluyen el uso de antipsicóticos, inhibidores de la acetilcolinesterasa y aquellas utilizadas en el manejo anestésico.

\section{Antipsicóticos típicos y atípicos}

El haloperidol (antipsicótico típico) fue evaluado contra placebo en pacientes sometidos a cirugía de fractura de cadera ${ }^{82}$, utilizando 0,5

Tabla 2. Factores de riesgo y medidas de prevención

\begin{tabular}{|ll|}
\hline Factor de riesgo & Medida preventiva \\
\hline Alteración visual - auditiva & Lentes ópticos-audífonos \\
\hline Alteración cognitiva & Reorientación frecuente \\
\hline Alteración funcional & Sacar al paciente de la cama y minimizar restricciones físicas \\
$\begin{array}{l}\text { Deshidratación-Alteración de electrolitos } \\
\text { plasmáticos }\end{array}$ & $\begin{array}{l}\text { Reconocimiento temprano y reposición vía oral idealmente. Restaurar equilibrio } \\
\text { electrolítico }\end{array}$ \\
Medicación de alto riesgo & $\begin{array}{l}\text { Minimizar uso de benzodiacepinas, antihistamínicos, anticolinérgicos y meperi- } \\
\text { dina. Minimizar las dosis y número de drogas }\end{array}$ \\
Deprivación de sueño & Mantener ciclo sueño-vigilia \\
\hline Dolor & Preferir terapia multimodal \\
Desnutrición & Suplementar ingesta calórica \\
latrogenia & $\begin{array}{l}\text { Asegurar tránsito intestinal y micción - kinesioterapia respiratoria - oxígeno } \\
\text { suplementario - transfusiones }\end{array}$ \\
\hline
\end{tabular}


mg c/8 h vía oral, disminuyendo la duración y severidad del delirium, pero sin impacto en su incidencia. Probablemente debido a que ambos grupos recibieron la consulta activa del equipo de geriatría. Otro estudio ${ }^{83}$ evaluó el uso profiláctico de 1,7 mg de haloperidol versus placebo, en pacientes sometidos a cirugía mayor no cardiaca, demostrando una menor incidencia de DPO en el grupo haloperidol. Esta disparidad en la incidencia de delirium respecto al primer estudio, podría deberse a los mayores niveles plasmáticos alcanzados al administrar el fármaco vía endovenosa.

Entre los antipsicóticos atípicos, el uso de risperidona ${ }^{84}$ y olanzapina han disminuido la incidencia de DPO. Sin embargo, cuando se utilizó olanzapina el delirium fue más severo y de mayor duración, descartándose como opción profiláctica $^{85}$.

Pese a estos estudios, el uso profiláctico de antipsicóticos aún no está recomendado.

\section{Inhibidores de la acetilcolinesterasa}

En la fisiopatología del delirium existe un déficit de acetilcolina en el sistema nervioso central. La administración de un fármaco capaz de aumentar sus niveles podría prevenir o tratar el delirium. Varios estudios han evaluado el donepezil ${ }^{86-88} \mathrm{y}$ la rivastigmina ${ }^{89,90}$ sin demostrar un rol positivo en la prevención, tratamiento o disminución de la severidad del delirium, pudiendo incluso tener una relación con el aumento de la mortalidad en pacientes de UCI, como en el caso de la rivastigmina ${ }^{90}$.

\section{Cuidado anestésico y sedación en UCI}

\section{Anestesia general versus anestesia regional}

Una de las preguntas que surge cuando un paciente se somete a una cirugía y presenta DPO es si la anestesia general fue el desencadenante de éste. Existen a la fecha una revisión sistemática ${ }^{91}$ y un meta-análisis ${ }^{92}$, los cuales concluyen que el uso de anestesia general no es un factor desencadenante de DPO. Por lo tanto, ¿qué otros factores influyen en la aparición de DPO en quienes reciben anestesia regional? La respuesta podría estar en el grado de sedación durante este tipo de anestesia ${ }^{93}$, concluyendo que su limitación se asociaría con un menor porcentaje de DPO.

\section{Alfa 2 agonista}

La dexmedetomedina ha demostrado tener una más baja incidencia de delirium al compararla con morfina ${ }^{94}$, haloperidol ${ }^{95}$, propofol $^{96}$, midazolam $^{96,97}$ y lorazepam ${ }^{98}$. Sin embargo, un reciente meta-análisis no logró demostrar un impacto positivo en la incidencia de delirium en el grupo dexmedetomedina ${ }^{99}$. Esta conclusión se debería a que no en todos los trabajos se evaluó como resultado primario la incidencia de delirium o no se utilizaron herramientas objetivas para su detección.

$\mathrm{Su}$ principal beneficio sobre los agentes de uso habitual para sedación estaría dado por la carencia de efectos anticolinérgicos, regulación del ciclo sueño vigilia y un menor requerimiento de opioides y agonistas $\mathrm{GABA}^{100-103}$, asociado también a una importante contención de $\operatorname{costos}^{104}$. Además su uso es útil en los casos de delirium refractario, considerando sus efectos adversos y requerimiento de monitorización.

Todos estos resultados alientan su uso en el futuro, necesitándose mayores estudios que lo sustenten.

\section{Opioides y analgésicos}

Tanto el dolor postoperatorio como su manejo están involucrados en la aparición de $\mathrm{DPO}^{105,106}$. El uso opioides debe ser juicioso y administrado en cantidad suficiente para un manejo óptimo del dolor con la menor incidencia de efectos adversos. Una revisión sistemática ${ }^{107}$ evaluó diferentes opioides, demostrando que sólo el uso de meperidina sería un factor precipitante para el desarrollo de DPO. Brouquet y cols. evaluaron el uso de tramadol (300 mg endovenosos al día en infusión continua) en pacientes sometidos a gran cirugía abdominal, demostrando ser el principal factor de riesgo de DPO. Por lo tanto, en pacientes de riesgo sería aconsejable administrar otros agentes analgésicos o proveer menores dosis a las administradas en este estudio ${ }^{108}$.

El uso de bloqueos regionales como método analgésico ha demostrado impactar favorablemente en la incidencia, severidad y duración del delirium $^{109,110}$.

\section{Tratamiento}

La evidencia que sustenta el tratamiento, es más escasa que la que soporta las medidas profilácticas. 
Las medidas de tratamiento también se pueden dividir en no farmacológicas y farmacológicas.

\section{Medidas no farmacológicas}

Idealmente realizada por un equipo multidisciplinario. Al parecer la efectividad de estas medidas tendría impacto en reducir la duración y severidad del delirium, así como los días de hospitalización ${ }^{111-113}$. Aunque no tengan respaldo suficiente basado en la evidencia, diversas guías clínicas las recomiendan ${ }^{114,115}$. Estas medidas son: descartar una complicación quirúrgica o médica, cuidado continuo de enfermería, asegurar nutrición e hidratación, movilización precoz, asegurar patrón de ciclo sueño-vigilia y manipulación del ambiente, reorientación (reloj, calendario, recordar el espacio-tiempo), evitar cambios excesivos de personal y minimizar alteraciones sensoriales (uso de lentes ópticos y audífonos $)^{116}$. Las restricciones físicas son cuestionables ya que pueden aumentar la agitación ${ }^{117,118}$, pero si ésta pone en riesgo al paciente (retiro de catéteres, tubo endotraqueal, caídas, etc.) se hacen necesarias ${ }^{119}$. Muy importante es el involucrar a la familia en el cuidado del paciente ${ }^{116}$, teniendo un impacto altamente positivo en su evolución ${ }^{119}$.

\section{Medidas farmacológicas}

No existe consenso acerca del tratamiento farmacológico ideal ${ }^{120,121}$. En la práctica, el manejo de delirium está basado en reporte de casos y series retrospectivas ${ }^{120,121}$. La Sociedad de Medicina Intensiva y el Colegio Americano de esta subespecialidad recomiendan el haloperidol para controlar la agitación y síntomas psicóticos ${ }^{122}$, sin embargo, el diagnóstico de delirium no es una indicación del uso de sedantes o antipsicóticos ${ }^{123}$. Lo que sí está claro, como primera medida, es corregir los factores médicos desencadenantes o agravantes del cuadro, así como optimizar el manejo preventivo ambiental. En cuanto a la terapia farmacológica, preferir la monoterapia, iniciar con la mínima dosis y ajustarla gradualmente ${ }^{124}$. La meta debe ser un paciente despierto, no agitado y libre de síntomas psicóticos.

Entre los antipsicóticos el más utilizado es el haloperidol ${ }^{125,126}$, recomendándose una dosis inicial de 0,5 a $1 \mathrm{mg}$ vía oral. En el manejo agudo del paciente agitado y combativo se recomienda su administración endovenosa, la cual se ha asociado a prolongaciones del segmento QT del electrocardiograma y desenlaces fatales ${ }^{127}$. En marzo de 2012, el Instituto de Salud Pública de Chile (ISP) recomendó evitar el uso de haloperidol endovenoso, limitando el tratamiento agudo de un paciente agitado. Esta recomendación cambió al mes siguiente, pudiendo usarse haloperidol endovenoso, pero asociado a monitoreo cardiaco, reapareciendo esta importante herramienta terapéutica. Se recomienda comenzar con haloperidol endovenoso con dosis de 0,5-1 mg y titular según efecto clínico, evaluando al paciente cada $20 \mathrm{~min}$ $y$, según necesidad, doblando la dosis previa. Al día siguiente de iniciada la terapia se sugiere evaluar el paso a vía enteral e ir disminuyendo en $50 \%$ la dosis total requerida en el día previo, con una duración del tratamiento, según evolución, entre 5-7 días. Los antisicóticos atípicos no han demostrado superioridad respecto al haloperidol en términos de eficacia ${ }^{122,128-130}$, sin embargo, presentarían un menor porcentaje de efectos secundarios. Además, el uso de estos agentes en población mayor con demencia se ha asociado a accidentes cerebrovasculares y muerte. Entre los más utilizados destaca: la quetiapina, recomendándose comenzar con dosis orales de 25 a $100 \mathrm{mg}$ al día, la risperidona, 0,5 a $1 \mathrm{mg}$ cada ocho a doce horas y la olanzapina, con dosis de 2,5 a $5 \mathrm{mg}$ al día en una o dos tomas. Si los mayores síntomas de agitación ocurren durante la noche, se recomienda que el uso de antipsicóticos sea entre las 21 y $23 \mathrm{~h}$.

Las benzodiacepinas no están recomendadas para el manejo del delirium ya que pueden aumentar el nivel de agitación o incluso empeorar su curso y duración ${ }^{127,131}$. Los únicos casos que recomiendan su uso son el delirium asociado a abstinencia de alcohol o benzodiacepinas, el delirium asociado a convulsiones y aquellos pacientes con contraindicación de antisicóticos (enfermedad de Parkinson, demencia por cuerpos de Lewy o el antecedente de síndrome neuroléptico maligno).

\section{Conclusiones}

El DPO es una complicación frecuente en el adulto mayor y su incidencia depende del tipo de cirugía. Su aparición puede significar un resultado adverso en el período perioperatorio inmediato y a largo plazo. Es importante su prevención y búsqueda diagnóstica activa, especialmente en 
aquellos pacientes con mayor riesgo de desarrollar esta complicación. En caso de diagnosticarse se deben continuar las medidas de prevención e implementar idealmente un manejo multidisciplinario.

\section{Referencias}

1. Jackson R, Strauss R, Howe N. El desafío del envejecimiento en América Latina, ONU 2009.

2. Silbert B, Evered L, Scott DA. Cognitive decline in the elderly: Is anaesthesia implicated? Best Practice \& Research Clinical Anaesthesiology 2011; 25: 379-93.

3. Sanders RD, Pandharipande PP, Davidson AJ, Ma D, Maze M. Anticipating and managing postoperative delirium and cognitive decline in adults. BMJ 2011; 343-8.

4. Diagnostic and Statistical Manual of Mental Disorders. 4th ed. Washington, DC: American Psychiatric Association; 1994.

5. Voyer P, Richard S, Doucet L, Carmichael PH. Detecting delirium and subsyndromal delirium using different diagnostic criteria among demented long-term care residents. J Am Med Dir Assoc 2009; 10 (3): 181-8.

6. Cole M, Mc Cusker J, Dendukuri N, Han L. The prognostic significance of subsyndromal delirium in elderly medical inpatients. J Am Geriatr Soc 2003; 51 (6): 75460.

7. Ouimet S, Riker R, Bergeron N, Cossette M, Kavanagh B, Skrobik Y. Subsyndromal delirium in the ICU: Evidence for a disease spectrum. Intensive Care Med 2007; 33: 1007-13.

8. Levkoff SE, Yang FM, Liptzin B. Delirium: the importance of subsyndromal states. Prim Psychiatry 2004; 11: 40-4.

9. Watt DF, Koziol K, Budding D. "Delirium and confusional states". In Disorders in Neuropsychiatry, eds C.A. Noggleand R.S. Dean (New York: Springer Publishing Company). 2012.

10. Dyer CB, Ashton CM, Teasdale TA. Postoperative delirium. A review of 80 primary data-collection studies. Arch Intern Med 1995; 155: 461-5.

11. Martínez-Velilla N, Alonso Bouzón C, Ripa Zazpe C, Sánchez-Ostiz R. Síndrome confusional agudo postoperatorio en el paciente anciano. Cir Esp 2012; 90 (2): 75-84.

12. Fong TG, Jones RN, Shi P, Marcantonio ER, Yap L, Rudolph JL, et al. Delirium accelerates cognitive decline in Alzheimer disease. Neurology 2009; 72: 1570-5.

13. Milbrandt EB, Deppen S, Harrison PL, Shintani AK, Speroff $\mathrm{T}$, et al. Costs associated with delirium in me- chanically ventilated patients. Crit Care Med 2004; 32 (4): 955-62.

14. Leslie DL, Marcantonio ER, Zhang Y, Leo-Summers L, Inouye SK. One-year health care costs associated with delirium in the elderly population. Arch Intern Med 2008; 168 (1): 27-32.

15. Rudolph JL, Jones RN, Rasmussen LS, Silverstein JH, Inouye SK, Marcantonio ER. Independent vascular and cognitive risk factors for postoperative delirium. Am J Med 2007; 120: 807-13.

16. Koebrugge B, Koek HL, van Wensen RJ, Dautzenberg PL, Bosscha K. Delirium after abdominal surgery at a surgical ward with a high standard of delirium care: incidence, risk factors and outcomes. Dig Surg 2009; 26: 63-8.

17. Rudolph JL, Jones RN, Levkoff SE, Rockett C, Inouye SK, Sellke FW, et al. Derivation and validation of a preoperative prediction rule for delirium after cardiac surgery. Circulation 2009; 119: 229-36.

18. Lundstrom M, Edlund A, Bucht G, Karlsson S, Gustafson Y. Dementia after delirium in patients with femoral neck fractures. J Am Geriatr Soc 2003; 51: 1002-6.

19. Bruce AJ, Ritchie CW, Blizard R, Lai R, Raven P. The incidence of delirium associated with orthopedic surgery: a meta-analytic review. Int Psychogeriatr 2007; 19: 197-214.

20. Deiner S, Silverstein JH. Postoperative delirium and cognitive dysfunction. Br J Anaesth 2009; 103 (Suppl 1): i41-6.

21. Takeuchi M, Takeuchi H, Fujisawa D, Miyajima K, Yoshimura K, Hashiguchi S, et al. Risk Factors of Postoperative Delirium in Patients with Esophageal Cancer. Ann Surg Oncol 2012; 19 (12): 3963-70.

22. Ansaloni L, Catena F, Chattat R, Fortuna D, Franceschi C, Mascitti P, et al. Risk factors and incidence of postoperative delirium in elderly patients after elective and emergency surgery. Br J Surg 2010; 97 (2): 273-80.

23. Kiely DK, Bergmann MA, Jones RN, Murphy KM, Orav EJ, Marcantonio ER. Characteristics associated with delirium persistence among newly admitted postacute facility patients. J Gerontol A Biol Sci Med Sci 2004; 59 (4): 344-9.

24. Cole MG, Ciampi A, Belzile E, Zhong L. Persistent delirium in older hospital patients: a systematic review of frequency and prognosis. Age Ageing 2009; 38 (1): 19-26.

25. Olin K, Eriksdotter-Jönhagen M, Jansson A, Herrington MK, Kristiansson M, Permert J. Postoperative delirium in elderly patients after major abdominal surgery. $\mathrm{Br} \mathrm{J}$ Surg 2005; 92 (12): 1559-64. 
26. Ganai S, Lee KF, Merrill A, Lee MH, Bellantonio S, Brennan $\mathrm{M}$, et al. Adverse outcomes of geriatric patients undergoing abdominal surgery who are at high risk for delirium. Arch Surg 2007; 142 (11): 1072-8.

27. Witlox J, Eurelings LSM, de Jonghe JFM, Kalisvaart KJ, Eikelenboom P, van Gool WA. Delirium in elderly patients and the risk of postdischarge mortality, institutionalization, and dementia: a meta-analysis. JAMA 2010; 304 (4): 443-51.

28. González M, Carrasco M. Delirium: marcador de estado del paciente geriátrico. Rev Esp Geriatr Gerontol 2008; 43 (Supl. 3): 38-41.

29. Ely EW, Gautam S, Margolin R, Francis J, May L, Speroff $\mathrm{T}$, et al. The impact of delirium in theintensive care unit on hospital length of stay. Intensive Care Med 2001; 27: 1892-900.

30. Ely EW, Shintani A, Truman B, Speroff T, Gordon SM, Harrell FE Jr, et al. Delirium as a predictor of mortality in mechanically ventilated patients in the intensive care unit. JAMA 2004; 291 (14): 1753-62.

31. Maldonado JR. Delirium in the acute care setting: characteristics, diagnosis and treatment. Crit Care Clin 2008; 24 (4): 657-722.

32. Liptzin B, Levkoff SE. An empirical study of delirium subtypes. Br J Psychiatry 1992; 161: 843-5.

33. Yang FM, Marcantonio ER, Inouye SK, Kiely DK, Rudolph JL, Fearing MA, et al. Phenomenological subtypes of delirium in older persons: patterns, prevalence, and prognosis. Psychosomatics 2009; 50: 248-54.

34. O'Keeffe ST, Lavan JN. Clinical significance of delirium subtypes in older people. Age Ageing 1999; 28: 115-9.

35. Meagher DJ, Leonard M, Donnelly S, Conroy M, Adamis D, Trzepacz PT. A longitudinal study of motor subtypes in delirium: relationship with other phenomenology, etiology, medication exposure and prognosis. J Psychosom Res 2011; 71 (6): 395-403.

36. Maldonado JR. Pathoetiological model of delirium: a comprehensive understanding of the neurobiology of delirium and an evidence-based approach to prevention and treatment. Crit Care Clin 2008; 24 (4): 789-856.

37. Broadhurst $\mathrm{C}$, Wilson K. Immunology of delirium: new opportunities for treatment and research. Br J Psychiatry 2001; 179: 288-9.

38. Cole MG. Delirium in elderly patients. Am J Geriatr Psychiatry 2004; 12 (1): 7-21.

39. Khan BA, Zawahiri M, Campbell NL, Boustani MA. Biomarkers for Delirium-A Review. J Am Geriatr Soc 2011; 59 Suppl 2: S256-61.

40. Marcantonio ER. Postoperative delirium: a 76-year-old woman with delirium following surgery. JAMA 2012; 308 (1): 73-81.
41. Hshieh TT, Fong TG, Marcantonio ER, Inouye SK. Cholinergic deficiency hypothesis in delirium: a synthesis of current evidence. J Gerontol A Biol Sci Med Sci 2008; 63 (7): 764-72.

42. Trzepacz PT. Is there a final common neural pathway in delirium? Focus on acetylcholine and dopamine. Semin Clin Neuropsychiatry 2000; 5 (2): 132-48.

43. Gaudreau JD, Gagnon P. Psychotogenic drugs and delirium pathogenesis: the central role of the thalamus. Med Hypotheses 2005; 64 (3): 471-5.

44. Cerejeira J, Nogueira V, Luís P, Vaz-Serra A, Mukaetova-Ladinska EB. The cholinergic system and inflammation: common pathways in delirium pathophysiology. J Am Geriatr Soc 2012; 60 (4): 669-75.

45. Colkesen Y, Giray S, Ozenli Y, Sezgin N, Coskun I. Relation of serum cortisol to delirium occurring after acute coronary syndromes. Am J Emerg Med 2013; 31 (1): 161-5.

46. Kudoh A, Takase H, Katagai H, Takazawa T. Postoperative interleukin-6 and cortisol concentrations in elderly patients with postoperative confusion. Neuroimmunomodulation 2005; 12 (1): 60-6.

47. McIntosh TK, Bush HL, Yeston NS, Grasberger R, Palter M, Aun F, et al. Beta-endorphin, cortisol and postoperative delirium: a preliminary report. Psychoneuroendocrinology 1985; 10 (3): 303-13.

48. Wong CL, Holroyd-Leduc J, Simel DL, Straus SE. Does this patient have delirium?: value of bedside instruments. JAMA 2010; 304: 779-86.

49. Inouye SK, van Dyck CH, Alessi CA, Balkin S, Siegal AP, Horwitz RI. Clarifying confusion: the confusion assessment method: a new method for detection of delirium. Ann Intern Med 1990; 113 (12): 941-8.

50. Wei LA, Fearing MA, Sternberg EJ, Inouye SK. The Confusión Assessment Method a systematic review of current usage. J Am Geriatr Soc 2008; 56 (5): 823-30.

51. Bruno JJ, Warren ML. Intensive care unit delirium. Crit Care Nurs Clin North Am 2010; 22: 161-78.

52. Ely EW, Margolin R, Francis J, May L, Truman B, Dittus $\mathrm{R}$, et al. Evaluation of delirium in critically ill patients: validation of the Confusion Assessment Method for the Intensive Care Unit (CAM-ICU). Crit Care Med 2001; 29: 1370-9.

53. Ely EW, Inouye SK, Bernard GR, Gordon S, Francis J, May L, et al. Delirium in mechanically ventilated patients: validity and reliability of the confusion assessment method for the intensive care unit (CAMICU). JAMA 2001; 286: 2703-10.

54. McNicoll L, Pisani MA, Ely EW, Gifford D, Inouye SK. Detection of delirium in the intensive care unit: comparison of confusion assessment method for the intensive 
care unit with confusion assessment method ratings. J Am Geriatr Soc 2005; 53: 495-500.

55. Plaschke K, von Haken R, Scholz M, Engelhardt R, Brobeil A, Martin E, et al. Comparison of the confusion assessment method for the intensive care unit (CAMICU) with the Intensive Care Delirium Screening Checklist (ICDSC) for delirium in critical care patients gives high agreement rate(s). Intensive Care Med 2008; 34: 431-6.

56. Van Eijk MMJ, van Marum RJ, Klijn IAM, de Wit N, Kesecioglu J, Slooter AJC. Comparison of delirium assessment tools in a mixed intensive care unit. Crit Care Med 2009; 37: 1881-5.

57. Luetz A, Heymann A, Radtke FM, Chenitir C, Neuhaus U, Nachtigall I, et al. Different assessment tools for intensive care unit delirium: which score to use? Crit Care Med 2010; 38: 409-18.

58. Greer N, Rossom R, Anderson P, MacDonald R, Tacklind J, Rutks I, Wilt TJ. Washington (DC): Department of Veterans Affairs (US); Delirium: Screening, Prevention, and Diagnosis - A Systematic Review of the Evidence [Internet]. 2011.

59. Hengjing Zou, Wei Mei, Jin Qiu, Yongsheng Li, Zhiguo Zhang, Yuke Tian, Chuanhan Zhang. Perceptions, attitudes and practices of postoperative delirium: A survey of anesthetists from Chinese university hospitals. AsiaPacific Psychiatry 2011; 3 (2): 67-72.

60. Ely EW, Stephens RK, Jackson JC, Thomason JW, Truman B, Gordon S, Dittus RS, Bernard GR. Current opinions regarding the importance, diagnosis, and management of delirium in the intensive care unit: a survey of 912 healthcare professionals. Crit Care Med 2004; 32 (1): 106-12.

61. Litaker D, Locala J, Franco K, Bronson DL, Tannous Z. Preoperative risk factors for postoperative delirium. Gen Hosp Psychiatry 2001; 23: 84-9.

62. Inouye SK, Viscoli CM, Horwitz RI, Hurst LD, Tinetti ME. A predictive model for delirium in hospitalized elderly medical patients based on admission characteristics. Ann Intern Med 1993; 119 (6): 474-81.

63. Kalisvaart KJ, Vreeswijk R, de Jonghe JF, van der Ploeg T, van Gool WA, Eikelenboom P. Risk factors and prediction of postoperative delirium in elderly hip-surgery patients: Implementation and validation of a medical risk factor model. J Am Geriatr Soc 2006; 54: 817-22.

64. Noimark D. Predicting the onset of delirium in the postoperative patient. Age Ageing 2009; 38: 368-73.

65. Galanakis P, Bickel H, Gradinger R, von Gumppenberg S, Forstl H. Acute confusional state in the elderly following hip surgery: incidence risk factors and complications. Int J Geriatr Psychiatry 2001; 16: 349-55.
66. Böhner H, Hummel TC, Habel U, Miller C, Reinbott S, Yang $Q$, et al. Predicting delirium after vascular surgery: a model based on pre- and intraoperative data. Ann Surg 2003; 238: 149-56.

67. Norkiene I, Ringaitiene D, Misiuriene I, Samalavicius R, Bubulis R, Baublys A, et al. Incidence and precipitating factors of delirium after coronary artery bypass grafting. Scand Cardiovasc J 2007; 41: 180-5.

68. Dasgupta M, Dumbrell AC. Preoperative risk assessment for delirium after noncardiac surgery: a systematic review. J Am Geriatr Soc 2006; 54: 1578-89.

69. Litaker D, Locala J, Franco K, Bronson DL, Tannous Z. Preoperative risk factors for post-operative delirium. Gen Hosp Psychiatr 2001; 23: 84-9.

70. Marcantonio ER, Goldman L, Mangione CM, Ludwig LE, Muraca B, et al. A clinical prediction rule for delirium after elective noncardiac surgery. JAMA 1994; 271: 134-9.

71. Galanakis P, Bickel H, Gradinger R, von Gumppenberg $\mathrm{S}$, Forstl $\mathrm{H}$. Acute confusional state in the elderly following hip surgery: incidence risk factors and complications. Int J Geriatr Psychiatry 2001; 16: 349-55.

72. Böhner H, Hummel TC, Habel U, Miller C, Reinbott S, Yang Q, et al. Predicting delirium after vascular surgery: a model based on pre- and intraoperative data. Ann Surg 2003; 238: 149-56.

73. Rubin FH, Neal K, Fenlon K, Hassan S, Inouye SK. Sustainability and scalability of the hospital elder life program at a community hospital. J Am Geriatr Soc 2011; 59: 359-65.

74. Inouye SK, Bogardus ST Jr, Charpentier PA, Leo-Summers L, Acampora D, Holford TR, et al. A multicomponent intervention to prevent delirium in hospitalized older patients. N Engl J Med 1999; 340 (9): 669-76.

75. Chen CCH, Lin MT, Tien YW, Yen CJ, Huang GH, Inouye SK. Modified hospitalelder life program: effects on abdominal surgery patients. J Am Coll Surg 2011; 213 (2): 245-52.

76. Marcantonio ER, Flacker JM, Wright RJ, Resnick NM. Reducing delirium after hip fracture: a randomized trial. J Am Geriatr Soc 2001; 49: 516-22.

77. Siddiqi N, House AO, Holmes JD. Occurrence and outcome of delirium in medical in-patients: a systematic literature review. Age Ageing 2006; 35: 350-64.

78. Vidán MT, Sánchez E, Alonso M, Montero B, Ortiz J, Serra JA. An intervention integrated into daily clinical practice reduces the incidence of delirium during hospitalization in elderly patients. J Am Geriatr Soc 2009; 57: 2029-36

79. Skrobik Y, Ahern S, Leblanc M, Marquis F, Awissi DK, Kavanagh BP. Protocolized intensive care unit mana- 
gement of analgesia, sedation, and delirium improves analgesia and subsyndromal delirium rates. Anesth Analg 2010; 111: 451-63.

80. Girard TD, Kress JP, Fuchs BD, Thomason JW, Schweickert WD, Pun BT, et al. Efficacy and safety of a paired sedation and ventilator weaning protocol for mechanically ventilated patients in intensive care (Awakening and Breathing Controlled trial): a randomized controlled trial. Lancet 2008; 371: 126-34.

81. Pandharipande P, Banerjee A, McGrane S, Ely EW. Liberation and animation for ventilated ICU patients: the ABCDE bundle for the back-end of critical care. Crit Care 2010; 14 (3): 157.

82. Kalisvaart KJ, de Jonghe JF, Bogaards MJ, Vreeswijk R, Egberts TC, Burger BJ, et al. Haloperidol prophylaxis for elderly hip-surgery patients at risk for delirium: a randomized placebo-controlled study. J Am Geriatr Soc 2005; 53 (10): 1658-66.

83. Wang W, Li HL, Wang DX, Zhu X, Li SL, Yao GQ, et al. Haloperidol prophylaxis decreases delirium incidence in elderly patients after noncardiac surgery: a randomized controlled trial. Crit Care Med 2012; 40 (3): 731-9.

84. Prakanrattana U, Prapaitrakool S. Efficacy of risperidone for prevention of postoperative delirium in cardiac surgery. Aging Clin Exp Res 2007; 19: 178-86.

85. Larsen KA, Kelly SE, Stern TA, Bode RH Jr, Price LL, Hunter DJ, et al. Administration of olanzapine to prevent postoperative delirium in elderly joint replacement patients: a randomized, controlled trial. Psychosomatics 2010; 51 (5): 409-18.

86. Liptzin B, Laki A, Garb JL, Fingeroth R, Krushell R. Donepezilin the prevention and treatment of post-surgical delirium. Am J Geriatr Psychiatry 2005; 13: 1100-6.

87. Sampson EL, Raven PR, Ndhlovu PN, Vallance A, Garlick N, Watts J, et al. A randomized, double-blind, placebo controlled trial of donepezil hydrochloride (Aricept) for reducing the incidence of postoperative delirium after elective total hip replacement. Int J Geriatr Psychiatry 2007; 22: 343-9.

88. Marcantonio ER, Palihnich K, Appleton P, Davis RB. Pilot randomized trial of donepezil hydrochloride for delirium after hip fracture. J Am Geriatr Soc 2011; 59 Suppl 2: S282-8.

89. Gamberini M, Bolliger D, Lurati Buse GA, Burkhart CS, Grapow M, Gagneux A, et al. Rivastigmine for the prevention of postoperative delirium in elderly patients undergoing elective cardiac surgery-a randomized controlled trial. Crit Care Med 2009; 37: 1762-8.

90. Van Eijk MM, Roes KC, Honing ML, Kuiper MA, Karakus $A$, van der Jagt $M$, et al. Effect of rivastigmine as an adjunct to usual care with haloperidol on duration of delirium and mortality in critically ill patients: a multicentre, double blind, placebo controlled randomised trial. Lancet 2010; 376: 1829-37.

91. Bryson GL, Wyand A. Evidence-based clinical update: general anesthesia and the risk of delirium and postoperative cognitive dysfunction. Can J Anesth 2006; 53: 669-77.

92. Mason SE, Storr AN, Ritchie CW. The impact of general and regional anesthesia on the incidence of postoperative cognitive dysfunction and postoperative delirium: A systematic review with metaanalysis. Journal of Alzheimer's Disease 2010; 22: S67-S79.

93. Sieber FE, Zakriya KJ, Gottschalk A, Blute MR, Lee $\mathrm{HB}$, Rosenberg PB, et al. Sedation depth during spinal anesthesia and the development of postoperative delirium in elderly patients undergoing hip fracture repair. Mayo Clin Proc 2010; 85 (1): 18-26.

94. Shehabi Y, Grant P, Wolfenden H, Hammond N, Bass F, Campbell M, et al. Prevalence of delirium with dexmedetomidine compared with morphine based therapy after cardiac surgery: a randomized controlled trial (Dexmedetomidine Compared to Morphine-DEXCOM Study). Anesthesiology 2009; 111 (5): 1075-84.

95. Reade MC, O'Sullivan K, Bates S, Goldsmith D, Ainslie WR, Bellomo R. Dexmedetomidine vs haloperidol in delirious, agitated, intubated patients: a randomised open-label trial. Crit Care 2009; 13: R75.

96. Ruokonen E, Parviainen I, Jakob SM, Nunes S, Kaukonen $\mathrm{M}$, Shepherd ST, et al. Dexmedetomidine versus propofol/midazolam for long-term sedation during mechanical ventilation. Intensive Care Med 2009; 35: 282-90.

97. Riker RR, Shehabi Y, Bokesch PM, Ceraso D, Wisemandle W, Koura F, et al. Dexmedetomidine vs midazolam for sedation of critically ill patients: a randomized trial. JAMA 2009; 301: 489-99.

98. Pandharipande PP, Sanders RD, Girard TD, McGrane S, Thompson JL, Shintani AK, et al. Effect of dexmedetomidine versus lorazepam on outcome in patients with sepsis: an a priori-designed analysis of the MENDS randomized controlled trial. Crit Care 2010; 14: R38.

99. Tan JA, Ho KM. Use of dexmedetomidine as a sedative and analgesic agent in critically ill adult patients: a metaanalysis. Intensive Care Med 2010; 36: 926-39.

100. Buttermann AE, Reid K, Maze M. Are cholinergic pathways involved in the anesthetic response to -2 agonists. Toxicol Lett 1998; 100-101: 17-22.

101. Hsu YW, Cortinez LI, Robertson KM, Keifer JC, SumPing ST, Moretti EW, et al. Dexmedetomidine pharmacodynamics: part I: crossover comparison of the respiratory effects of dexmedetomidine and remifentanil 
in healthy volunteers. Anesthesiology 2004; 101 (5): 1066-76.

102. Maldonado JR, Wysong A, van der Starre PJ, Block T, Miller C, Reitz BA: Dexmedetomidine and the reduction of postoperative delirium after cardiac surgery. Psychosomatics 2009; 50 (3): 206-17.

103. Nelson LE, Lu J, Guo T, Saper CB, Franks NP, Maze M. The $\alpha 2$ adrenoceptor agonist dexmedetomidine converges on an endogenous sleep-promoting pathway to exert its sedative effects. Anesthesiology 2003; 98 (2): 428-36.

104. Dasta JF, Kane-Gill SL, Pencina M, Shehabi Y, Bokesch $\mathrm{P}$, Wisemandle $\mathrm{W}$, et al. A cost minimization analysis of dexmedetomidine compared with midazolam for longterm sedation in the intensive care unit. Crit Care Med 2010; 38 (2): 497-503.

105. Lynch EP, Lazor MA, Gellis JE, Orav J, Goldman L, Marcantonio ER. The impact of postoperative pain on the development of postoperative delirium. Anesth Analg 1998; 86: 781-5.

106. Burkhart CS, Dell-Kuster S, Gamberini M, Moeckli A, Grapow M, Filipovic M, et al. Modifiable and nonmodifiable risk factors for postoperative delirium after cardiac surgery with cardiopulmonary bypass. J Cardiothorac Vasc Anesth 2010; 24: 555-9.

107. Fong HK, Sands LP, Leung JM. The role of postoperative analgesia in delirium and cognitive decline in elderly patients: a systematic review. Anesth Analg 2006; 102: 1255-66.

108. Brouquet A, Cudennec T, Benoist S, Moulias S, Beauchet A, Penna C, et al. Impaired mobility, ASA status and administration of tramadol are risk factors for postoperative delirium in patients aged 75 years or more after major abdominal surgery. Ann Surg 2010; 251 (4): 759-65.

109. Mouzopoulos G, Vasiliadis G, Lasanianos N, Nikolaras G, Morakis E, Kaminaris M. Fascia iliaca block prophylaxis for hip fracture patients at risk for delirium: a randomized placebo-controlled study. J Orthop Traumatol 2009; 10 (3): 127-33.

110. Del Rosario E, Esteve N, Sernandez MJ, Batet C, Aguilar JL. Does femoral nerve analgesia impact the development of postoperative delirium in the elderly? A retrospective investigation. Acute Pain (2008) 10, 59-64.

111. Lundström M, Edlund A, Karlsson S, Brännström B, Bucht G, Gustafson Y. A multifactorial intervention program reduces the duration of delirium, length of hospitalization, and mortality in delirious patients. J Am Geriatr Soc 2005; 53 (4): 622-8.

112. Pitkä lä KH, Laurila JV, Strandberg TE, Tilvis RS. Mul- ticomponent geriatric intervention for elderly inpatients with delirium: a randomized, controlled trial. J Gerontol A Biol Sci Med Sci 2006; 61 (2): 176-81.

113. Lundström M, Olofsson B, Stenvall M, Karlsson S, Nyberg L, Englund U, et al. Postoperative delirium in old patients with femoral neck fracture: a randomized intervention study. Aging Clin Exp Res 2007; 19 (3): 178-86.

114. Tropea J, Slee JA, Brand CA, Gray L, Snell T. Clinical practice guidelines for the management of delirium in older people in Australia. Australas J Ageing 2008; 27: 150-6.

115. Tahir TA, Morgan E, Eeles E. NICE guideline: evidence for pharmacological treatment of delirium. J Psychosom Res 2011; 70: 197-8.

116. NICE. (2010). Delirium: Diagnosis, Prevention and Management. (Clinical Guideline 103). National Institute for Health and Clinical Excellence. Available at: www. nice.org.uk/CG103.

117. Inouye SK. Delirium in older persons. N Engl J Med 2006; 354 (11): 1157-65.

118. Young J, Inouye SK. Delirium in older people. BMJ 2007; 334 (7598): 842-6.

119. Marcantonio ER. In the clinic. Delirium. Ann Intern Med 2011; 154 (11): ITC6-1, ITC6-2, ITC6-3, ITC6-4, ITC6-5, ITC6-6, ITC6-7, ITC6-8, ITC6-9, ITC6-10, ITC6-11, ITC6-12, ITC6-13, ITC6-14, ITC6-15; quiz ITC6-16.

120. Fearing MA, Inouye SK. Delirium. FOCUS 2009; 7: $53-$ 63.

121. Bourne RS, Tahir TA, Borthwick M, Sampson EL. Drug treatment of delirium: past, present and future. J Psychosom Res 2008; 65 (3): 273-82.

122. Lonergan E, Britton AM, Luxenberg J, Wyller T. Antipsychotics for delirium. Cochrane Database Syst Rev 2007; (2): CD005594.

123. Seitz DP, Gill SS, van Zyl LT. Antipsychotics in the treatment of delirium: a systematic review. J Clin Psychiatry 2007; 68 (1): 11-21.

124. Jacobi J, Fraser GL, Coursin DB, Riker RR, Fontaine D, Wittbrodt ET, et al. Clinical practice guidelines for the sustained use of sedatives and analgesics in the critically ill adult. Crit Care Med 2002; 30 (1): 119-41.

125. Flaherty JH, Gonzales JP, Dong B. Antipsychotics in the treatment of delirium in older hospitalized adults: a systematic review. J Am Geriatr Soc 2011; 59 Suppl 2: S269-76.

126. Rivera R, Antognini JF. Perioperative drug therapy in elderly patients. Anesthesiology 2009; 110: 1176-81.

127. Breitbart W, Marotta R, Platt MM, Weisman H, Derevenco $\mathrm{M}$, Grau C, et al. A double-blind trial of haloperi- 
dol, chlorpromazine, and lorazepam in the treatment of delirium in hospitalized AIDS patients. Am J Psychiatry 1996; 153 (2): 231-7.

128. Hu H, Deng W, Yang H, Liu Y. Olanzapine and haloperidol for senile delirium: a randomized controlled observation. Chin J Clin Rehab 2006. 10: 188-90.

129. Meyer-Massetti C, Cheng CM, Sharpe BA, Meier CR, Guglielmo BJ. The FDA extended warning for intrave- nous haloperidol and torsades de pointes: how should institutions respond? J Hosp Med 2010; 5: E8-16.

130. Han CS, Kim YK. A double-blind trial of risperidone and haloperidol for the treatment of delirium. Psychosomatics 2004; 45 (4): 297-301.

131. Lonergan E, Luxenberg J, Areosa Sastre A. Benzodiazepines for delirium. Cochrane Database Syst Rev 2009. CD006379. 